\title{
Quantitative Evaluation for the Level of Intellectual Property Protection in China
}

\author{
Liping $\mathbf{X u}^{1,2}$ \\ ${ }^{1}$ University of Shanghai for Science and Technology, Shanghai, China \\ ${ }^{2}$ Shanghai Publishing and Printing College, Shanghai, China \\ Email: mlbsabc@sina.com
}

How to cite this paper: Xu, L.P. (2017) Quantitative Evaluation for the Level of Intellectual Property Protection in China. Open Journal of Social Sciences, 5, 120-129. https://doi.org/10.4236/jss.2017.54011

Received: February 22, 2017

Accepted: April 18, 2017

Published: April 21, 2017

Copyright $\odot 2017$ by author and Scientific Research Publishing Inc. This work is licensed under the Creative Commons Attribution International License (CC BY 4.0).

http://creativecommons.org/licenses/by/4.0/

\begin{abstract}
Making a review for the quantitative evaluation research of Intellectual Property Protection Level's in china, we get the research status, research perspective and the research method of the study from the review. After analyzing the existed problem and deficiency of those studies, we set forth the prerequisite of Intellectual Property Protection Level's study, and some new study thoughts, which are based on performance theory and value chain theory. We suggest a new study method should be used in the quantitative evaluation study of intellectual property protection level, which is a comprehensive evaluation method based on experts information. In the end, the principle of constructing parameter system will be elaborated, which will be used in quantitative evaluation study of intellectual property protection.
\end{abstract}

\section{Keywords}

Intellectual Property Protection, Ginarte-Park Method, Value Chain, Comprehensive Evaluation Method

\section{Introduction}

Intellectual property protection has been an international policy concern. It is a legal confirmation to the right, which comes from intellectual activities in the field of science, technology, culture, art, etc. It is a complex problem, which is related with economic, science and technology, culture, education and others. More and more scholars have engaged in the research of intellectual property protection since China's accession to the WTO. There are different opinions about the protection level of intellectual property in China. Some scholars believe that Chinese existing intellectual property protection level is higher than what can be overwhelming, which is not conducive to economic development. Some scholars think that Chinese intellectual property protection level is lower. It is not suitable 
to rapid economic growth, and adverse to the national development.

Foreign scholars' research shows choosing appropriate strategy of intellectual property protection is helpful to economic grow [1] [2]. So, we must make clear of intellectual property protection level and do some adjust to the protection strategy. It is a key point to quantitative evaluation for the level of intellectual property protection. In this review, we make careful analysis comparison of previous research on quantitative evaluation of intellectual property rights in China, point out the shortage of existing research on the basis of summarizing research ideas and research methods. We put forward a new research perspective and research method, combined with the existing scholar's research and full consideration of actual economic and social conditions. West rive to make quantitative evaluation research of the intellectual property protection level has more realistic guiding significance that can give some specific advice to corresponding industry development or give some efficiency suggestion to adjust policy, but not give theory description only.

\section{The Quantitative Evaluation Research Status}

\subsection{Research Review}

After intellectual property protection being the basic framework of the international trade in WTO, the research related to it quickly became a hot topic. The protection issue of intellectual property is complicated, which affected by such factors as the legislative, judicial and law enforcement. It is more difficult to directly evaluate the level of intellectual property protection. [3] At present, most scholars have conducted qualitative studies or analysis of the theoretical model [4], because of lacking of quantitative measurement method. After joining the WTO, Chinese scholars have begun to use quantitative analysis methods to measure the level of intellectual property protection.

In 2005, Yuxiong Han and Huaizu Li pointed out that intellectual property protection level cannot be accurately measured by using the Rapp-Rozek method or Ginarte-Park method in China. They put forward a revised method by adding parameters related law enforcement variables to Ginarte-Park method, and apply it to measure the level of intellectual property protection level [3]. In 2008, Chunming $\mathrm{Xu}$ and Xiaoguang Shan revised the Ginarte-Park method by adding intensity of law enforcement as a parameter, and evaluate intellectual property protection level of China from 1985 to 2004. They pointed that intellectual property protection level will be affected by five key factors, such as judicial protection level, administrative protection level, economic development level, the social public consciousness and international supervision [5]. Hui Peng, Jiejing Yao has adopted similar approach to evaluate the IP protection level of 58 countries [4]. Xuebing Shen and Jia Liu put forwards that that the economic development level, degree of governed by law and enforcement of law are the three main factors to influent the intensity of actual IP protection, after measuring IP protection level from of 1995 to 2007, which results between that getting by G-P method and Han's method [4]. Xue-bing Dong believe less strict protection of 
intellectual property will more advantageous to our country economic growth in a short term. He get it from experiment result [4]. Hefa Song, Yuguang Li and Wan Qudo the experiment to respectively measure four kinds of ability, including intellectual property creation ability, intellectual property application ability, intellectual property protection ability and intellectual property management ability. They set up a parameters system, which includes 20 parameters. Research shows it is important to encourage intellectual property creation ability [6].

Since 2013, researches about IP protection have improved. Jing Yang, Xuezhong Zhu's research covered a wide field, concluding patent protection, copyright protection, trademark protection and geographical indication protection. Research objects are more comprehensive than previous research, which make research results more scientific and valid. They pointed out that high strength of the intellectual property protection in developing countries can lead to limited domestic policy system, the problems such as system establishment and operation cost increase. It is very important to do intellectual property protection of quantitative research. We should explore system establishment on the basis of protection economy, law and social influence with objective evaluation of intellectual property rights [7]. In 2013 YingZhan, revised the method of GinartePark by adding law enforcement indexes such as national intellectual property rights protection implementation index, law enforcement index of global rule of law and PC software piracy rate. He evaluated the influence of economic development level on IP protection in the world 122 countries. He judged whether the level of intellectual property rights is adapted to the current level of economic development and pointed out that China's legislative level is higher and the intellectual property protection level corresponded to the current economic developing level. [4] In 2012, Mei-ling Yang finished the evaluation index system of enterprise's intellectual property management research. [8] She put the four procedures of intellectual property rights into the concrete implementation of the enterprise to build the evaluation index system in order to improve the enterprise value maximization. [9] In 2012, HualinTan, Junqiang Zhang finished the evaluation research of judicial protection of intellectual property rights. They determined the evaluated objects of judicial protection of intellectual property rights and selected the key indicators of the performance evaluation. [10] In 2013, Lv Min, Ya-bin Zhang in the study of the actual intensity measurement for the protection of intellectual property, with the entropy method to determine the weighting parameters. They measured the actual strength for the protection of intellectual property in China from 1985 to 2010. They pointed out that the government strengthened the construction of related intellectual property law system, participated in the international intellectual property rules and other measures, can obviously accelerate and promote the protection of intellectual property rights [11]. In 2014, Chunlin Chen, Yanqiu Song and Yunting Wang evaluated the performance of intellectual property management. They took intellectual property rights management of government funding research project as the research object. They set up the evaluation index system with the laws and regulations. They 
proposed a fuzzy comprehensive evaluation model based on expert opinion. They determined the evaluation index system with the expert opinion [12]. Sun He quantified and analyzed the law enforcement level of intellectual property of our country. They used the normalization method and the grey system model on data processing and pointed out that the administrative law enforcement protection is the important means to realize the protection of intellectual property rights protection from the level of judicial protection of intellectual property and administrative protection law enforcement level, social and enterprise and individual protection level [13].

\subsection{The Characteristics of the Existing Research}

In recent years, many Chinese scholars have carried out quantitative evaluation research of intellectual property protection in the field of law, economics, science and technology. In the field of law, the study focuses on quantitative evaluate relevant legislation ability, judicial protection ability and IP protection law enforcement ability. Most scholars applied the revised G-P method to measure the intellectual property protection level, emphasizes IP protection system security and order, social values and public interests. Researcher in the field of science and technology Focus on the development and application of advanced science and technology. They research all kinds of intellectual property protection technology and method.

The commonly used research methods includes: 1) the questionnaire method; 2) Ginerate-Park method; 3) the information processing method. Ginerate-Park method is an evaluation method for the strength of intellectual property legislation, is not for the protection level of intellectual property. In some studies, scholars set the equal weights for different lever parameters, ignoring its different impact degree on the intellectual property protection, which will probably make results less scientific, less valid. In recent studies, some scholars have been aware of the shortage, gradually introduced the data analysis method, such as fuzzy mathematics theory and entropy theory to set parameter weight.

\section{The Limitation of the Existing Research}

1) The regional differences were not been considered. People's protection consciousness varies from different economic conditions. If not considering regional economic differences, but blindly implement the intellectual property policies, it will be frustrated. In China, there is unbalanced development and the economic condition is difference in various regions. So, regional differences must be considered in the research.

2) Applicable Conditions of research is not been illustrated. Intellectual property protection is a complicated issue related with the legislative, judicial, economy, technology, culture, education, social environment and etc. [4]. In the study, Most scholars choose these factors, which relevant to the economic, technical, and social environment, as parameters that have an effect on enforcement ability. The results of that study will be limited by the condition of research en- 
vironment. So, applicable Conditions of research should be illustrated. In reality, if condition of the legislative level, economic condition and social environment does not meet the applicable environment, the application of research is meaningless. I think that study of intellectual property is valuable only in specific conditions: 1) the complete legal system; 2) the higher degree of governed by law; 3) the stability of the economic level; 4) adaptable level of law enforcement to the current economic.

3) Main types of intellectual property were not involved comprehensively in study, the results of study has limitations. At present, most study about intellectual property protection mainly do macro-research to make clear that effect of Intellectual property protection on whole society. Patent data is chosen to represent the whole intellectual property category, while intellectual property includes patent, trademark, copyright. There are limitations to research only using patent data. Foreign scholars have set about to conducted studies according to the categories of intellectual property [13].

4) Proxy variable for same parameter is not same in different study. After choosing parameters, proxy variable will be used to replace the parameter values, such as the number of lawyers to measure the judicial protection level, legislative years measure whether the legal system is complete, the per capita GDP to measure economic development level (some studies using urban per capita disposable income to measure economic development level), the trademark administrative enforcement cases measure administrative law enforcement efficiency (some measured by the copyright administrative authorities investigate case level administrative law enforcement efficiency) [11] [14] [15]. That would lead to the results are inconsistent.

5) Lack of corresponding evaluation. The existing evaluation study of intellectual property protection focus on evaluating a country's overall strength of intellectual property protection, [7] there is less targeted evaluation. There is no quantitative evaluation research have been conducted to the administrative department of industry, industry and different policy.

\section{The New Perspective of Quantitative Evaluation Research of Intellectual Property Rights Protection}

With the development of the quantitative evaluation research of intellectual property protection, many scholars put forward the new idea. In order to break through the bottleneck of the present research, it is imperative to introduce new perspective and new method to research.

\subsection{Perspective Based on the Theory of the Performance}

Performance reflects the efficiency of organization in complete or exact fulfillment of the terms of plan for achieving goals [16]. Performance targets of the intellectual property protection is to attach importance to on both social public interests and the balance between fairness and efficiency, while intellectual property right were protected effectively and properly. The object of perfor- 
mance evaluation is a series of activities, which can be evaluated, in four process of intellectual property work.

The performance evaluation parameters are diverse, and evaluation criterion is dynamic, but not static [17]. The parameters are difference in various stages of development [16]. We select the performance evaluation parameters from the objects and users of four processes in intellectual property work. It will make evaluation parameter set comprehensive.

We do the quantitative evaluation research of intellectual property protection will be helpful to make the intellectual property system healthy, orderly and sustainable.

\subsection{Comprehensive Evaluation Idea Based on Value Chain}

In 1985, Michael porter of Harvard University introduced analysis method based on value chain in book: competitive advantage. [18] The enterprise and relevant unit seemed to be a whole body, it create the same value. [8] In recent years, foreign scholars have done the research of intellectual property protection using that theory based on supply chain management [19]. In China, Heng Tang, Yang Zhang and Jinyan Chai was proposed to do intellectual property research based on value chain. They qualitatively expounded and analyzed the theory [8], but no quantitative evaluation.

Intellectual property value chain is the combination of Michael porter's value chain theory and the intellectual property management [20]. The value of intellectual property including creator's value and social value, it covers four process of intellectual property work: intellectual property creation, intellectual property utilization and intellectual property protection, and intellectual property management. It organically combines all processes together, and it makes a more complete intellectual property research. comprehensive evaluation idea based on value chain make four process connection and integration effectively to raise their ability, maximizing the value of intellectual property.

\subsection{Commercial Perspective Evaluation, Comprehensive Evaluation by Using Parameters Covered Field of Technology, Economy and Law}

In China, the research of intellectual property protection mainly concentrated on legal in the field of law, concentrated on new technology in the field of science \& technology, or concentrated on economic income distribution in the field of economics. There is no combination research of law, economy and technology. It is significant to build analysis frame work with "technologyeconomy-aw", study the effect of intellectual property of on market structure and competition from the view of commerce. It can be more comprehensive quantitative evaluation of intellectual property protection level.

\subsection{Classification Evaluation According to the Kind of Intellectual Property}

There are three important content of Intellectual property: the patent, trademark 
and copyright. They have the following characteristics in China: 1) administrative department of intellectual property is varied from different categories of intellectual property. There is difference in aspect of law enforcement strength [14]. 2) private rights and public rights is different, resulting in different social value; 3) protection consciousness is different between different categories of intellectual property; 4) corresponding value chain is different, and the objects in the value chain is also different.

In the existing research of intellectual property protection, the patent data has been used to instead of three kind of intellectual property, it lead to that result has a lot of restrictions. Especially in the environment of culture industry rapid development, copyright of digital publishing is very important. I think the research results should be more effective in realistic guiding significance, if the research have been done in accordance with the patent, trademark, copyright (copyright), respectively.

\section{New Research Methods of Quantitative Evaluation}

\subsection{Information Technology Related with E-Government Performance Evaluation}

Chu Zhang introduces the concept of performance evaluation to quantitative evaluation research of intellectual property protection first time in the paper: "On the Constitution of the Performance Evaluation System of Intellectual Property Protection". He introduced the foreign government performance control target system, and put forward three kinds of e-government control goal system: COBIT system, FEA system and EAF system. In his research, Chu Zhang points out that those evaluation model system have consult value for evaluation of intellectual property protection in our country [19]. EAF system has strong Indian aboriginality, so we temporarily not to consider this approach.

COBIT is internationally recognized standards for management and control of security and information technology, it is the integrated information control system, in which the performance evaluation is an important component. COBIT system is helpful to quantitative evaluation of intellectual property protection. We can do the research follow steps: 1) defining the high-level performance evaluation goal of intellectual property protection; 2) describing the evaluation criteria; 3 ) in a series of activities, defining evaluation goals in detail according to the high-level goal; 4) give scores to parameters according to the evaluation criteria; 5) applying three kinds of assessment tools to research: the key success factors, key performance indicators and key target index.

\subsection{System Comprehensive Evaluation Method Based on Expert Selection}

In recent years, scholars have applied comprehensive evaluation methods to quantitative evaluation research of intellectual property protection level, such as fuzzy theory, gray model, entropy value method and so on [9]. There is broader research idea and better research methods than before. At present research, most 
scholars resolve problem in a certain way, which lead to inconsistent evaluate conclusion because of different methods, or random error caused by single quantitative method. Seeking for the new evaluation method is necessary to make the research effectively, On the premise of fully recognize evaluation objects, external environment and the evaluation requirements. Professor Qian Xuesen proposed a method of processing complex giant system, which is comprehensive integration method from qualitative to the quantitative thinking. It is be used to combination evaluation research based on the method set. Under the guidance of the basic evaluation principles, several methods will be concentrated extracted from the set of basic evaluation method according to certain standards and rules, and then the object will be evaluated by those methods, which will eliminate the system error and random error caused by single method. At the same time, it can solve the inconsistency problem evaluation conclusion. In the study, if we can study with combined method based on expert suggestion, the research conclusion will be more scientific and more effective.

\section{Rules of Building a Quantitative Evaluation Index Set}

It is an importance step for research of intellectual property protection quantitative evaluation to building a relevant evaluation index set. There are some advices about choosing parameter, it follows.

Index source: index selected exist in the process of intellectual property, it contains four aspects: intellectual property creation, intellectual property application, intellectual property protection and intellectual property management. The system is not complete if one of the four aspects is absent [6].

The range of data: we need experiment data corresponding to index, which reflect the real situation. China joined in the WTO in 2001. After 2001, economic condition, social environment, degree of legislation and law enforcement has met the essential condition for the research of intellectual property protection in China. Making use of data from 2001 to 2015 to do research, the research results will have more scientific and realistic significance for the future work.

The basic principle of index selection: it is key point for the guarantee of results is scientific and validity to establish the index system. It is complex and difficult, we should follow principles: 1) the index set should containboth quantitative parameters and qualitative parameters; 2) the scientific principle; 3) comprehensive, systemic principle; 4) operational principle; 5) the principle of stability.

\section{Conclusions}

Quantitative research of intellectual property protection came up in the 1980's. Scholars adopted various quantitative or qualitative analysis methods to measure the level of intellectual property protection. The most famous is the Rapp-Rozek method and Ginarte-Park method. In China, people get the concept of intellectual property later than other countries. People lack protection consciousness of private property. Until 2001, after the accession to the WTO, Chinese scholars 
actively have begun to do research on the protection of intellectual property, but the method is limited, mostly is revised method but not technique innovation.

Intellectual property, which is core elements of international competitiveness and resource of national development strategic, has become an important means of development and competition in many countries. View from the angle of economics and management, it would have more practical value to do research of intellectual property protection based on the industrial chain. Intellectual property protection issue is complex, related with many field. Research on intellectual property protection should be system, comprehensive.

In China, economic development is different from different region. The quantitative evaluation study of intellectual property protection should fully consider the actual situation of unbalanced development in various regions. According to industry characteristics and intellectual property categories, targeted research will be better. The process of research needs to follow certain principles: firstly, choose the better research perspectives and target orientation; secondly, establishing a comprehensive \& systematic index system, and get the effective data that is the best response to actual situation; Last, choosing the appropriate research methods. That will ensure research process scientific and research conclusion effectiveness.

\section{References}

[1] Kim, Y.K., Lee, K., Park, W.G. and Choo, K. (2012) Appropriate Intellectual Property Protection and Economic Growth in Countries at Different Levels of Development. Research Policy, 41, 358-375.

[2] Gu, Z. and Shen, Y. (2015) Intellectual Property Rights Protection, Technology Innovation and Technology Transfer: From the Perspective of Developing Countries. Industrial Economics Research, No. 3, 474-493.

[3] Han, Y. and Li, H. (2005) Quantitative Analysis for Intellectual Property Protection Level of China. Studies in Science of Science, 23, 377-382. (In Chinese)

[4] Zhang, Y. (2013) Empirical Research on the Protection Level of Intellectual Property Right in China-International Compare and the Appropriateness Evaluation. Studies in Science of Science, 31, 1347-1354. (In Chinese)

[5] Xu, C.-M. and Shan, X.-G. (2008) Constructing of the Index System and Verification for the Intensity of Intellectual Property Protection in China. Studies in Science of Science, 26, 715-723. (In Chinese)

[6] Song, H., Li, Y. and Qu, W. (2013) The Intellectual Property Capacity Measurement Indicator System and Method Based on Equivalent Relationship of Kinds of Intellectual Property and Kinds of Research Institute. Studies in Science of Science, 31, 1826-1834. (In Chinese)

[7] Yang, J. and Zhu, X.-Z. (2013) The Index System of FTA Intellectual Property Strength and Measurement. Studies in Science of Science, 31, 883-891. (In Chinese)

[8] Tang, H. and Zhang, Y. (2013) A Study of the Enterprise Intellectual Property Standardization Management Based on the Value Chain. Science and Technology Management Research, No. 15, 175-180. (In Chinese)

[9] Yang, M.-L. (2012) The Evaluation Index System Research of High-Tech Enterprise Intellectual Property Management. Thesis, Guangdong University of Technology, Guangzhou. (in Chinese) 
[10] Tan, H. and Zhang, J. (2012) Research on Performance Evaluation of the Judicial Protection of Intellectual Property. Journal of Social Sciences, No. 5, 109-116. (In Chinese)

[11] Lv, M. and Zhang, Y. (2013) The Strength Measurement for Intellectual Property Protection in China. Science \& Technology Progress and Policy, 30, 113-118. (In Chinese)

[12] Chen, J., Song, Y., Wang, Y., et al. (2014) Evaluation for Performance of Intellectual Property Management: A Fuzzy Comprehensive Evaluation Model Based on the Specialists' Opinion Selection. China Science \& Technology Resources Review, 46, 15-22. (In Chinese)

[13] Park, W.G. (2008) International Patent Protection: 1960-2005. Research Policy, 37, 761-766.

[14] Sun, H. (2015) Measurement and Analysis of the Level of Law Enforcement of the Intellectual Property Protection in China. Studies in Science of Science, 33, 13721380. (In Chinese)

[15] Zhao, S.-M. (2003) The Conflict and Coordination between Information Resources Sharing and Intellectual Property Protection. Nanning, Guangxi University. (In Chinese)

[16] Zhang, C., Bai, J.-Y. and Dai, C. (2011) On the Constitution of the Performance Evaluation System of Intellectual Property Protection. Journal of Shandong Teachers' University (Humanities and Social Sciences), 56, 120-127. (In Chinese)

[17] Thomas, D.J. and Griffin, P.M. (1996) Coordinated Supply Chain Management. European Journal of Operation Research, 94, 1-15.

[18] Tsay, A. and Nahmias, S. (2000) Modeling Supply Chain Contracts-A Review. In: Quantitative Models for Supply Chain Management, Kluwer Academic Publisher, Amsterdam, 299-366.

[19] Lawrence, D., Letmathe, P. and Uebe-Emden, N. (2016) Supply Chain Management Practices and Intellectual Property Protection in China: Perceptions of Mittelstand managers. International Journal of Operations \& Production Management, 36, 135163. https://doi.org/10.1108/IJOPM-12-2013-0526

[20] Chai, J.-Y. (2009) The Cultivation of Intellectual Property Competitive Advantage of Enterprises Based on Value Chain. Science \& Technology Progress and Policy, 26, 53-56. (In Chinese)

Submit or recommend next manuscript to SCIRP and we will provide best service for you:

Accepting pre-submission inquiries through Email, Facebook, LinkedIn, Twitter, etc. A wide selection of journals (inclusive of 9 subjects, more than 200 journals)

Providing 24-hour high-quality service

User-friendly online submission system

Fair and swift peer-review system

Efficient typesetting and proofreading procedure

Display of the result of downloads and visits, as well as the number of cited articles

Maximum dissemination of your research work

Submit your manuscript at: http://papersubmission.scirp.org/

Or contact jss@scirp.org 